\title{
WEAK LENSING AND THE SLOAN DIGITAL SKY SURVEY
}

\author{
Wide Area Weak Lensing
}

\author{
ALBERT STEBBINS, TIM MCKAY, AND JOSHUA A. FRIEMAN \\ Fermi National Accelerator Laboratory \\ Box 500, Batavia IL 60510, USA
}

\begin{abstract}
While the strategy for the first applications of weak lensing has been to "go deep" it is equally interesting to use one's telescope time to instead "go wide". The Sloan Survey (SDSS) provides a natural framework for a very wide area weak lensing survey.
\end{abstract}

Probing of the mass distribution using the distortion of galaxy image shapes by the intervening gravitational field this mass produces is a powerful new technique for probing cosmological structure (Valdes et al. 1983, Tyson et al. 1990, Miralda-Escudé 1991, Blandford et al. 1991, Kaiser 1992). The "weak lensing" technique will no doubt become one of the standard probes, on par with galaxy redshift surveys and maps of CMBR anisotropies. Except for small areas on the sky near distant rich clusters or very near galaxies, the image distortion is expected to be small and weak lensing is the appropriate technique. One must average over many galaxies to obtain a significant detection of the small image distortion; typically by measuring correlations in galaxy position-angles and thus the shear. Deep imaging is extremely useful as it allows one to get accurate estimates of the shapes of large numbers of background galaxies in the relatively small field of view of most telescopes. If one fails to go deep one can identify fewer background galaxies and, in any case, one obtains only accurate shape information for the brighter, larger galaxies. However even with moderately deep images one can, in principle, use the weak lensing technique to infer the foreground mass distributions. If the the number of galaxies per unit area for which one has accurate shape information is small then one should survey a larger area to obtain a significant signal.

The first successful applications of the weak lensing (Tyson et al. 1990, Smail et al. 1994, Fahlman et al. 1995) has naturally been to take deep images of galaxies behind rich clusters where the shear is large, and perhaps 
more importantly, where one has a fairly good idea what one expects to find. Attempts have also been made to detect shear in the field (i.e. a direction not associated with a particular galaxy concentration), but without any definitive detection (Mould et al. 1994). When looking at the field one can expect to find contributions to image distortions from mass at various distances along the line-of-sight. While it would be useful to study the statistical properties of the shear at a given depth, one will, in the end, want to chart how the shear varies with depth. By understanding the variation with depth one can learn about the radial distribution of densities along different lines-of-sight. This can tell us something about the evolution of the density field and in particular about cosmological parameters such as $\Omega$ and $\Lambda$; as well as allow one to construct a crude map of the mass distribution. The latter application is particularly interesting as it will allow one to compare the mass distribution with the better studied nearby galaxy distribution (Villumsen \& Gould 1994). Thus even if one had a very deep survey of galaxy image shapes one would want to study the dependence of shear with depth and in effect look at less deep surveys. The study of shear at $z \sim 0.1-0.4$ is interesting in its own right!

Any imaging survey of the sky is implicitly measuring the shapes of the galaxies it is able to detect. As long as the combination of depth and area of the survey are large enough to obtain a sufficient $\mathrm{S} / \mathrm{N}$ one can in principle use this for weak lensing. One's calculation of depth must take into account the accuracy with which one is able measure the galaxy shapes. However it is generally true that one does not loose much by even relatively large random errors in the galaxy shapes. This is because the intrinsic non-circularness of the true projected galaxy shapes introduces random uncertainties in the inferred shear and one would have to make fairly large measurement errors to significantly add to these uncertainties. The true galactic position-angles are (assumed) random and therefore by using a sufficiently large number of galaxies one can reduce both the intrinsic and measurement uncertainties if they are random. However if uncorrected measurement errors are correlated between different galaxies one may never reach an acceptable $\mathrm{S} / \mathrm{N}$. Since the deeper the survey is the larger the signal will be, the requirement to control these systematic errors is less. It is not clear to what level one can reduce systematic errors and it is thus not clear how shallow a survey one could use for weak lensing studies.

The Sloan Digital Sky Survey (SDSS) (Kent 1994) is a prime example of a large imaging survey on which one may "piggy-back" a weak lensing program (Villumsen \& Gould 1994). Perhaps the most publicized aspect of the SDSS is a redshift survey of $10^{6}$ galaxies. To obtain the redshift targets the SDSS will image $1 / 4$ of the sky in 5 colors, mostly around the North Galactic Cap, identifying galaxies in the North down to a nominal magni- 
tude limit of $r^{\prime}<23.1$, and going to 25.1 in parts of the Southern survey. This will yield a catalog of $\sim 5 \times 10^{7}$ galaxy images. One does not really need the galaxies near the limiting magnitude to obtain a significant weak lensing signal and in this sense the SDSS can expect to do much better than a marginal detection. The multi-color photometry will be extremely useful for weak lensing as we expect to determine galaxy redshifts to $\Delta z \sim 0.04$ photometrically (Szalay 1995). With this redshift information one can map the shear as a function of distance. This allows one to better localize the mass distribution as a function of radius and make more of a direct comparison of the mass and galaxy distributions. Of course the SDSS redshift survey gives exactly the galaxy distribution one would want to compare to the mass distribution determined via weak lensing from the imaging survey.

In Villumsen and Gould (1994) it was estimated that nearby clusters and their extended halos would dominate the shear field measured by the SDSS. To illustrate some of the above comments in this regard let us consider the mean shear given by a model cluster with radial density profile

$$
\rho(r)=\frac{3 v_{1}^{2} r_{\mathrm{t}}^{2}}{2 \pi G\left(r^{2}+r_{\mathrm{c}}^{2}\right)\left(r^{2}+r_{\mathrm{t}}^{2}\right)} \quad r_{\mathrm{t}} \geq r_{\mathrm{c}}
$$

which is a kind of truncated non-singular isothermal sphere. For this profile the image shear as a function of angle, $\alpha$, from the cluster center is

$$
\gamma(\alpha)=6 \pi \frac{v_{1}^{2}}{c^{2}} \frac{\beta}{\alpha} \frac{\alpha_{\mathrm{t}}{ }^{2}}{{\alpha_{\mathrm{t}}{ }^{2}-{\alpha_{\mathrm{c}}}^{2}}^{2}}\left(2 \frac{\alpha_{\mathrm{t}}-\alpha_{\mathrm{c}}}{\alpha}-\frac{\alpha^{2}+2{\alpha_{\mathrm{t}}}^{2}}{\alpha \sqrt{\alpha^{2}+{\alpha_{\mathrm{t}}}^{2}}}+\frac{\alpha^{2}+2{\alpha_{\mathrm{c}}}^{2}}{\alpha \sqrt{\alpha^{2}+{\alpha_{\mathrm{c}}}^{2}}}\right) .
$$

Here $\alpha_{\mathrm{c}}$ and $\alpha_{\mathrm{t}}$ are the angles subtended by $r_{\mathrm{c}}$ and $r_{\mathrm{t}}$ at the distance of the cluster. The distances of the galaxies whose shear is measured comes into the factor $\beta$. If most of galaxies are much further away than the cluster then $\beta \approx 1$ while if most of the galaxies are at a distance comparable to or less than that of the cluster then $\beta$ may be much less than unity since many of the galaxies will be in front of the cluster and not sheared at all or not far enough behind the cluster to receive the full amount of shear.

The shear around a given cluster is maximized a few core radii from the center, while the maximal shear varies roughly proportional to $z$ until the cluster distance approaches the depth of the survey. One never finds large shear too close to the cluster center and for more nearby clusters one must look very far from the center to maximize the shear. Figure 1 illustrates that for shallow surveys one is most sensitive to nearby structures. Note that a disk radius of $5^{\prime}$ does a good job of maximizing the shear over a broad range of cluster redshifts and limiting magnitudes. Wider area coverage yields a larger signal only for $z \lesssim 0.2$ clusters. Of course a large signal is of no use unless one has sufficient galaxy numbers to detect it. In Figure 2 we see 

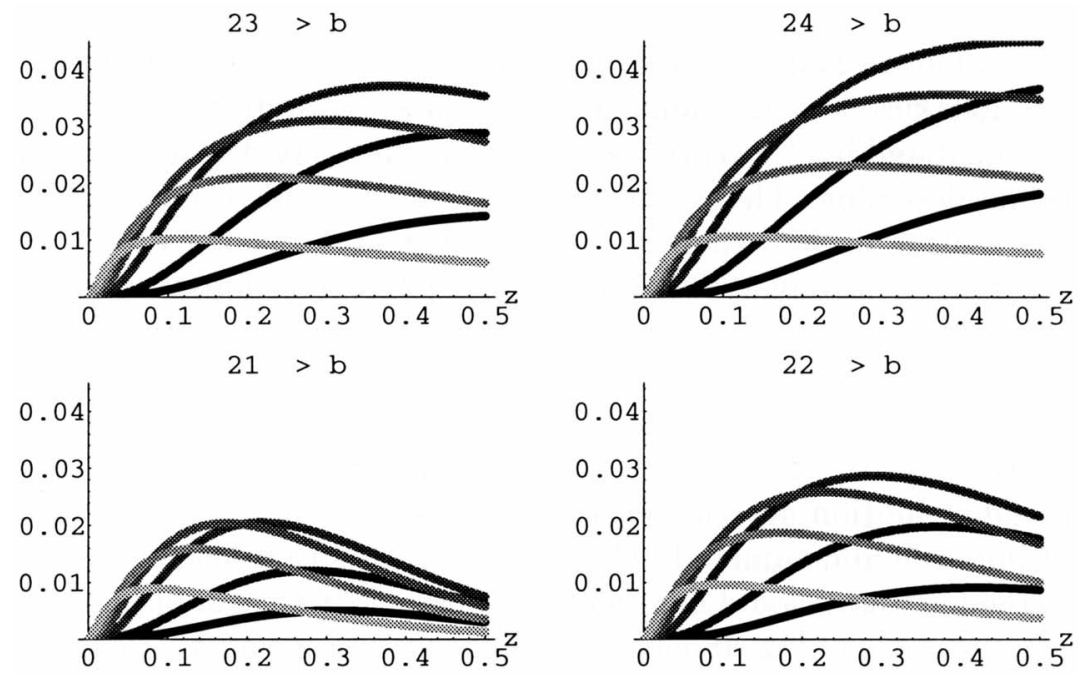

Figure 1. Plotted is the mean tangential shear in a disk on the sky centered on a model cluster vs. the redshift of the cluster. The different curves represent different disk radii: from black to light gray the radii are $1^{\prime}, 2^{\prime}, 5^{\prime}, 10^{\prime}, 20^{\prime}, 50^{\prime}$. The different plots are for different limiting $b$ magnitudes as labeled. Here we assume a Schechter luminosity function with $\phi_{*}=0.014(h / \mathrm{Mpc})^{3}, \alpha=-0.97$, and $M_{*}=-19.5$ corresponding to $b_{J}$ magnitudes. The model cluster has $1 \mathrm{~d}$ velocity dispersion $v_{1}=800 \mathrm{~km} / \mathrm{sec}$, core radius $r_{\mathrm{c}}=250 h^{-1} \mathrm{kpc}$, and truncation radius $r_{\mathrm{t}}=3 h^{-1} \mathrm{Mpc}$.

that the available $\mathrm{S} / \mathrm{N}$ is indeed significantly higher for deeper surveys but, with large enough area coverage, can be much larger than unity even for very shallow surveys. For extremely low redshift clusters one must survey very large areas to obtain significant signal. Yet even for $b<21$ one can in principle obtain a significant signal from a $z=0.03$ cluster, like Coma, if one is able to survey $\sim 1^{\circ 2}$. Note however that this would require keeping systematics well below the $1 \%$ level.

Given the low tolerance for systematic errors it is important to have a good handle on how well one is determining the shear. Besides simulations and comparison with better (i.e. HST) data, one can also use an internal check of one's data. To do this, take one's measured ellipticities and rotate their position-angle by $45^{\circ}$. Then use one's favorite reconstruction technique to estimate the surface density from the rotated data. The surface density one obtains should be consistent with zero up to the noise from the random galaxy orientations and known measurement errors; and from effects due to the boundary of one's sample. If not, one probably has discovered some systematic problems with one's method. The mathematics behind this is as follows. One is trying to estimate the shear tensor, $\gamma_{a b}$. Such a 2-d symmetric traceless tensor field can be decomposed into its 

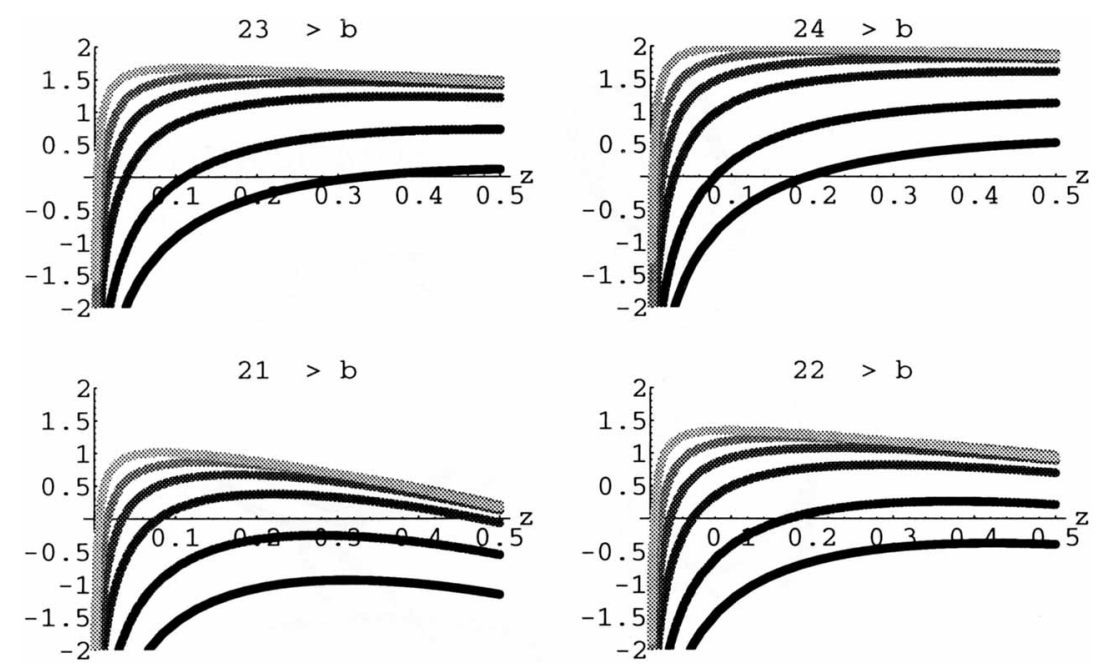

Figure 2. Plotted is $\log _{10} \mathrm{~S} / \mathrm{N}$ for measuring the amplitude of the shear for clusters as in Figure 1. The noise is assumed dominated by the finite number of non-round galaxies in the sample assumed to have rms ellipticity 0.3 .

scalar and pseudo-scalar parts

$$
\gamma_{\mathrm{s}}=\nabla^{-2} \gamma_{a b, a b} \quad \gamma_{\mathrm{p}}=\nabla^{-2} \epsilon_{a c} \gamma_{a b, b c} \quad \epsilon_{a b}=\left(\begin{array}{rr}
0 & -1 \\
1 & 0
\end{array}\right)
$$

which is analogous to decomposing a vector into its curl and a curl-free parts. For weak lensing $\gamma_{\mathbf{s}}$ is just proportional to the weighted surface density while for gravitationally induced shear from non-relativistic matter $\gamma_{\mathrm{p}}=0$ since the shear is derived from a potential. Multiplying the shear tensor by $\epsilon_{a b}$ is the same as rotating the position-angle of the shear by $45^{\circ}$, so one obtains the above result. The two components, $\gamma_{\mathrm{s}}$ and $\gamma_{\mathrm{p}}$, are so similar that most sources of noise and error will contribute equally to both, while the true signal will contribute only to $\gamma_{\mathrm{s}}$. Thus it is probably fair to believe one's results only to the extent that, on average, $\left|\gamma_{s}\right|>\left|\gamma_{p}\right|$. Kaiser and Tyson report that they have used similar methods.

At this writing the SDSS telescope is not yet operational and hence it is difficult to know how it will perform in practice. To address this issue the authors have begun an observational program with a telescope at the SDSS site, the ARC 3.5m telescope, using the Fermilab Drift Scan Camera (DSC) which is similar, if much smaller, than the SDSS camera. We have not yet reduced the level of systematics to the point which would make the SDSS Northern survey useful for weak lensing, but are confident that significant improvements will be made. The SDSS collaboration is in the process of comparing DSC data in the Sloan colors and at the Sloan depth 


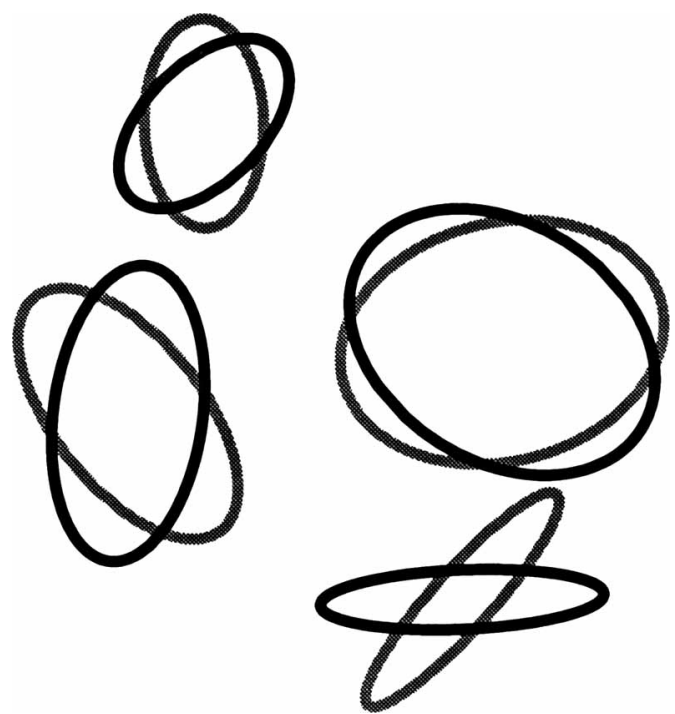

Figure 3. By rotating the measured ellipticities (black) by $45^{\circ}$ and then using these rotated ellipticities (gray) to reconstruct the surface density one constructs a realization of the same size as the error in the reconstructed surface density.

with deep HST WFPC-2 data. This will be extremely useful for gauging the accuracy of shear measurements that can be expected from the SDSS. If everything works well, the weak lensing data from the SDSS northern survey will be one of its major achievements. In any case we certainly do expect that the deeper SDSS southern survey will yield useful information from weak lensing studies.

Acknowledgements: This work was supported by the DOE and NASA grant \# NAG-5-2788.

\section{References}

Blandford, R., Saust, A., Brainerd, T., \& Villumsen, J., 1991, MNRAS, 251, 600

Fahlman, G., Kaiser, N., Squires, G., \& Woods, D., 1995, ApJ, 437, 56

Kaiser, N., 1992, ApJ, 388, 272

Kent, S., 1994, Ap \& Space Sci, 217, 27

Miralda-Escudé, J., 1991, ApJ, 380, 1

Mould, J., et al., 1994, MNRAS, 271, 56

Smail, I., Ellis, R., \& Fitchett, M., 1994, MNRAS, 270, 245

Szalay, A., 1995, private communication

Tyson, J., Valdes, F., \& Wenk, R., 1990, ApJL, 349, L1

Valdes, F., Tyson, J., \& Jarvis, J., 1983, ApJ, 271, 431

Villumsen, J., \& Gould, A., 1994, ApJL, 428, L45 